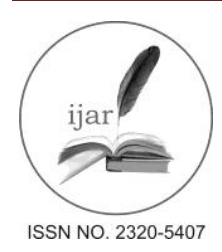

Journal homepage: http://www.journalijar.com

Journal DOI: 10.21474/IJAR01

INTERNATIONAL JOURNAL

OF ADVANCED RESEARCH

RESEARCH ARTICLE

\title{
A STUDY ON LOCATION-BASED ROUTING PROTOCOL FOR WSN
}

\section{Pavithra M.N ${ }^{1}$, Chinnaswamy C.N² .}

1. PG student, Department of IS\&E, The National institute of engineering, Mysore, Karnataka, India.

2. Associate Professor, The National institute of engineering, Mysore, Karnataka, India.

\section{Manuscript Info}

\section{Manuscript History:}

Received: 10 February 2016

Final Accepted: 22 March 2016

Published Online: April 2016

Key words:

Location based routing protocol,

GEF, GEAR, MECN, SMECN.

*Corresponding Author

Pavithra M.N

\begin{abstract}
A wireless sensor network (WSN) is a wireless network formed by distributed autonomous devices using sensors to oversee physical or environmental conditions. Wireless sensor networks consist of independent small-size Sensor nodes. Each sensor node collects data and sends it to different destinations. . In Wireless sensor networks, the sensor nodes have a limited transmission range as well as processing, energy resources and storage capabilities. The routing protocols for wireless sensor networks maintain the routes in the network, which might be depending on the application and network architecture and ensure reliable communication under these conditions. In this topic we explain location based routing protocols for Wireless sensor networks. In location-based protocols, address the location of sensor node. Location information about sensor nodes is required for sensor networks to calculate the distance between a pair of nodes so that energy consumption can be estimated. We present location-aware routing protocols for Wireless sensor networks.
\end{abstract}

Copy Right, IJAR, 2016, All rights reserved.

\section{Introduction:-}

A wireless Sensor Network consists of distinctively distributed nodes called sensors node that monitor physical and environmental conditions, such as pressure or temperature at different locations. In WSN have unique characteristics such as limited battery life for sensor nodes, computation, and communication capabilities. The characteristics of wireless sensor networks need a more effective approach for data forwarding and processing. In WSN, the sensor nodes have a restricted transmission range, and their handling and storage capacities and their energy assets are additionally constrained. Routing protocols for wireless sensor networks are maintaining the routes in the network and have to make sure reliable multi-hop communication under these conditions.

The rest of paper is organized as follows: section 1 present the design issues of routing protocols, section 2 describe routing protocol for WSNs. section 3 explain the classification location based routing protocol that include GAF, GEAR, SMECN, MECN and their advantages and disadvantages, section 4 concludes.

\section{Design issues of routing protocols:-}

WSNs were initially used by military applications. More recently used in the civilian application domain of wireless sensor networks such as environmental monitoring, air traffic controller, video surveillance, industrial and manufacturing automation, healthcare, smart home etc. These WSNs may consist of composite stationary or mobile sensor nodes, the design of routing protocols for WSNs it faces some challenging because of several network constraints.

The design challenges in sensor networks involve the following main aspects.

\section{a) Fault Tolerance:-}

The performance the sensor network should not affect the because of the failure. Some sensor nodes may fail or be blocked due to have physical damage or environmental interference. Path repair is desired in routing protocols 
when a path break is detected. Fault tolerance is the facilities to maintain sensor system functionalities with no interference because of sensor node disappointments.

\section{b) Scalability:-}

The numbers of sensor nodes deployed in wireless sensor network. The number of sensor nodes can be increased or decrease it depend on the requirement of the application. Wireless sensor network should accept the new one and coordinate them with existing nodes.

\section{c) Production Costs:-}

The sensor networks consist of a large number of sensor nodes, the cost of a single node is very important to validate the overall cost of the networks so the cost of each sensor node has to be kept low.

\section{d) Hardware design:-}

Because of the vitality compel in sensor system we ought to be more watchful While planning any equipment of sensor system, Hardware, for example, power unit, handling unit, small scale controller, and correspondence unit in a manner that it expends less vitality.

\section{e) Power Consumption:-}

The sensor hub lifetime emphatically relies upon battery life. Sensor hubs are outfitted with constrained force source $(<0.5 \mathrm{Ah} 1.2 \mathrm{~V})$. Power utilization can allot to three useful areas: detecting, correspondence, and information preparing, each of which requires advancement. Steering in sensor organize the multi-hop steering will devour less vitality than direct correspondence. Be that as it may, multi-hop steering presents noteworthy overhead for topology administration and medium access control. Direct steering would perform all around ok if every one of the hubs were near the sink.

\section{f) Quality of Service (QoS):-}

The quality of service means the capability of providing a level of services to its user within a period of time. Most of the application in WSNs is based on time (e.g. military application). Quality service is difficult in wsn because the constantly changing network topology. Many a time routing in sensor networks needs to sacrifice energy efficiency to meet delivery requirements.

\section{g) Node Deployment:-}

A node sending is relied on upon application and influences the execution of the directing convention. The association is either deterministic or self-sorting out. In deterministic circumstances, the sensors are physically put and information is directed through pre-decided ways. In self arranging frameworks, the sensor nodes are scattered haphazardly making a base in an Ad-hoc way. On that base, the position of the sink or the bunch head is additionally urgent as far as vitality effectiveness and execution. At the point when the circulation of nodes is not uniform, ideal setting of bunch head turns into a problem that needs to be addressed to empower vitality productive system operation.

\section{h) Data Aggregation/Fusion:-}

Data aggregation is that the gathering the information from multiple detector nodes. And additionally eliminate the redundant transmission and process it and providing amalgamated info to the bottom station. As computation would be less energy overwhelming than communication, substantial energy savings will be obtained through knowledge aggregation. This system has been accustomed reach energy potency and traffic optimization during a variety of routing protocols.

\section{Routing protocol for WSNs [5]:-}

WSNs area unit quite giant and that we will classify the routing protocol for WSNs in many various ways that. Routing protocols area unit classified as node central, data-centric, location-based (geo-centric) and QoS based mostly routing protocols. Data-centric routing the sink sends inquiries to bound districts and sits tight for data from the sensors settled inside of the hand-picked locales. Since data is being requested through queries, attribute based mostly naming is critical to indicate the properties of data. Here information is sometimes transmitted from each device node inside the preparation region with vital redundancy. Location info is acclimated enhance the performance of routing and to deliver new sorts of services. QoS based mostly routing protocols information delivery magnitude relation, latency and energy consumption area unit in the main thought-about. To urge an honest 
QoS (Quality of Service), the routing protocols should possess a lot of information delivery magnitude relation, less latency and less energy consumption. Node central routing or gradable routing protocol the goal of gradual routing is to manage the energy consumption of WNs expeditiously by establishing multihop communication inside a selected cluster.

Routing protocols also can be classified supported reactive or proactive. A proactive protocol sets up routing methods and states before there's a requirement for routing traffic. Methods are maintained even there's no traffic flow at that point. In the reactive routing protocol, routing actions are activated once there's data to be sent and dispersed to different nodes. Here methods are set up on demand once queries are initiated. Routing protocols are classified supported destination-initiated or source-initiated. A supply-initiated protocol sets up the routing methods upon the demand of the source node, and ranging from the supply node. Here supply advertises the info once accessible and initiates the info delivery.

A destination initiated protocol, initiates path setup from a destination node. Routing protocols are classified based mostly sensing element specification. Some WSNs comprises homogeneous nodes, whereas some comprises heterogeneous nodes. In view of this we can order the conventions whether they are working on a flat topology or on a hierarchal topology. In Flat routing protocols all nodes within the network area unit treated equally. Once a node must send knowledge, it's going to notice a route consisting of many hops to the sink.

A hierarchical routing protocol may be a natural approach to require for heterogeneous networks where a percentage of the node is more effective than alternate ones. The hierarchy doesn't invariably depend upon the ability of nodes. In hierarchical (Clustering) protocols completely different nodes are classified to make clusters and information from nodes happiness to one cluster is combined (aggregated). The agglomeration protocols have many benefits like scalable, energy economical find routes and straightforward to manage.

The network layer of sensing element networks contributes for routing in it. It's designed in keeping with the subsequent principles:

* Power potency is usually necessary.

* Sensor networks area unit principally knowledge central.

* Data aggregation is practicable only if it doesn't hamper the cooperative efforts of the sensing element nodes.

- An ideal sensing element network has attributed based mostly addressing and placement awareness.

In this paper explain location based routing protocol for WSN. In location-based protocols, address the location of sensor node. Location data for sensor nodes is required for sensor network to compute the separation between two specific nodes so that energy utilization can be evaluated. We present location-aware routing protocols for WSNs. We present mainly four location aware protocol Geographic Adaptive Fidelity (GAF), Geographic and EnergyAware Routing (GEAR), Minimum Energy Communication Network (MECN), Small Minimum-Energy Communication Network (SMECN).

WSNs are very huge and we can order the steering convention for WSNs in a wide range of ways. Routing protocols are classified as data-centric, node centric, location-based (geo-centric) and QoS based routing protocols.

\section{Data-centric routing:-}

The sink sends queries to sure regions and waits for information from the sensors situated within the selected regions. Considering data is being requested through queries, quality based naming is important to specify the properties of information. Here information is typically transmitted from each sensor node inside of the organization area with significant redundancy.

Example protocols: SPIN, Directed Diffusion, Rumour Routing, COUGAR, Gradient- Based Routing, ACQUIRE, Information-Directed Routing, Energy-aware Routing, Information-Directed Routing, Quorum-Based Information Dissemination, Home Agent Based Information Dissemination, EAD.

\section{Location aware routing:-}

Location information can be used to improve the performance of routing and to provide new types of services. Before that Nodes ensure that geographical region.

Example protocols: GAF, GEAR, MECN, SMECN, Span, BVGF, TBF, GeRaF. 
QOS based routing: - The data delivery path is computed using available resources in the network along with the QoS requirement for the particular application. QOS requirement are data delivery ratio, latency, and energy consumption.

Example protocols: SPEED, Energy-aware routing, SAR.

\section{Node centric routing:-}

Node centric is also called as hierarchal routing protocols are to deal with the energy utilization of WNs effectively by setting up multihop communication within a particular cluster.

Example Protocols: LEACH, HEED, TEEN, APTEEN, PEGASIS.

\section{Location based routing protocol:-}

In location-based protocols [13], Location information for sensor nodes is required for sensor networks for route discovery, data forwarding, and maintenance of sensor nodes on sensor network. To estimate energy consumption of network is to calculate the distance between two particular nodes and that helps in reducing energy consumption, and optimizing whole network.

\section{Geographic adaptive fidelity (GAF):}

GAF [4] (Energy aware routing protocol) is used for WSNs it's primarily proposed for MENETs. The configuration of GAF is motivated based on an energy model it deals with energy utilization because of the gathering and transmission of packets additionally in idle or listening time when the radio of the sensor is on to identify the presence of entering packets. While keeping a steady level of routing fidelity GAF turning off unnecessary sensors. In GAF, sensor field is separated into grid squares and each sensor utilizes its area data, which can be given by GPS or other area frameworks, to associate itself with a specific grid in which it lives..This sort of affiliation is abused by GAF to distinguish the sensors that are proportionate from the point of view of packet sending. As shown in Figure 3.1.a, the GAF state transition diagram o has three states, namely, discovery, active, and sleeping. In the sleeping state for the purpose of energy saving it offend its radio .In the discovery state, inside the equal grid to examine about other sensors the sensor exchanges discovery messages. In the active state, to inform equivalent sensors about its state the sensor periodically broadcasts its discovery message.

GAF aims to expend the lifetime of network by achieving a state where every grid one and only active sensor taking into account sensor positioning guidelines. The positioning of sensors depends on their leftover energy levels. The time spent in every of those states depends on the utility numerous factor which include sensor mobility and its needs.

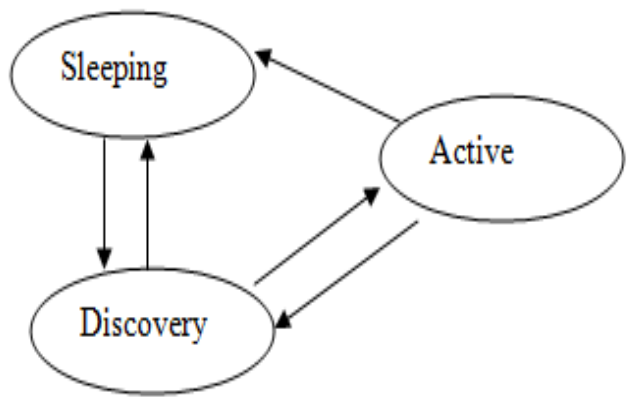

Fig. 3.1.a: State transition diagram of GAF.

GAF [4] is implemented each for non-mobility (GAF-basic) and mobility (GAF-mobility model) of nodes. Discern three.1.b suggests an example of fixed zoning that may be utilized in sensor networks just like the only proposed in. The fixed clusters in are decided on to be equal and square. The choice of the rectangular size is dependent on the specified transmitting electricity and the communiqué path. A vertical and horizontal conversation is guaranteed to occur if the sign travels a distance of $\mathrm{a}=\mathrm{r} / \sqrt{5}$ chosen such that any two sensor nodes in adjacent vertical or horizontal clusters can communicate immediately. 


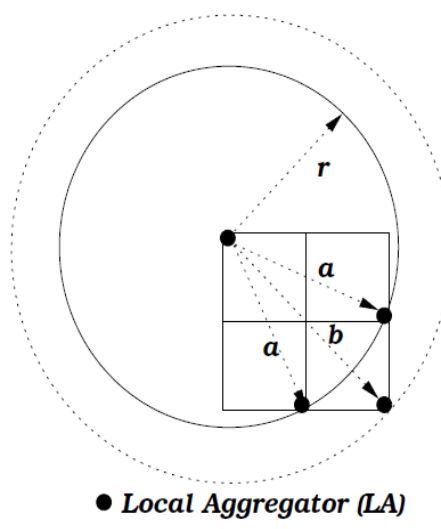

Fig 3.1.b: shows an example of fixed zoning in sensor network.

For a diagonal communique to appear, the sign has to span a distance of $b=r / 2 \sqrt{2}$. the issue is the way to schedule roles for the nodes to act as cluster heads. A cluster head can ask the sensor nodes in its cluster to replace on and begin amassing statistics if it senses an item. Then, cluster head is accountable for receiving uncooked data from different nodes in its cluster and forward it to the BS. The sensor nodes can realize their locations using GPS cards that are unbelievable with the present day generation. GAF strives to hold the community connected by means of preserving a representative node continually in lively mode for each place on its digital grid. Simulation consequences show that GAF performs as a minimum as well as a ordinary ad hoc routing protocol in terms of latency and packet loss and will increase the lifetime of the community by using saving strength. even though GAF is a area-based protocol, it could additionally be considered as a hierarchical protocol, where the clusters are based totally on geographic location. For every particular grid place, a representative node acts as the leader to transmit the information to different nodes.

\section{Geographic and energy-aware routing (gear):-}

GEAR [13] is an energy-efficient routing protocol proposed for routing queries to target area in a sensor discipline, In GEAR, the sensors are presupposed to have localization hardware ready, for example, a GPS unit or a localization machine in order that they recognize their current positions. Furthermore, the sensors are aware about their residual strength in addition to the locations and residual power of every of their neighbours. GEAR makes use of energy aware heuristics which might be primarily based on geographical statistics to pick out sensors to direction a packet in the direction of its vacation spot region. Then, GEAR uses a recursive geographic forwarding algorithm to disseminate the packet in the goal area.

The important thing is to limit rather than sending the interests to the whole network the number of interests in direct Diffusion by only considering a certain region, through doing this, GEAR can preserve greater energy than directed diffusion. In GEAR Each node carry an estimated cost of reaching destination through its neighbours. The cost is estimated using residual energy and distance to destination. A hole takes place when a node does no longer have any closer neighbour to the target place than itself. The learned cost is a refinement of the estimated cost that accounts for routing around holes in the network. if there are not any holes, the estimated cost is same to the learned cost. The found out cost is propagated one hop lower back every time a packet reaches the target in order that path setup for subsequent packet can be adjusted.

\section{Minimum energy communication network (MECN):}

MECN is a location-based protocol [13] which attempts to set up and maintain a path in the sense of energy efficiency for achieving minimum energy consumption in a given communication network. It is self-configuring protocol that keeps up system availability disregarding sensor mobility .It is compute sub network with minimum power from sensor to the sink node so is called minimum power topology. It can deployment new sensor or dynamically adopt node failure. This protocol has two phases based on the position of sensors on a plane namely, a) enclosure graph construction and b) cost distribution. The first phase enclosure graph construction, An enclosure graph $\mathrm{G}$ is a directed graph that vertex represent all the sensor nodes and edges set is represent path between every pair( $\mathrm{u}, \mathrm{v})$ of sensors nodes that are connected in G, edges between the sensors and the neighbours located in their enclosure regions. Minimum-energy path between $\mathrm{U}$ and $\mathrm{V}$, one that allows data to be transmitted among all the 
paths between $\mathrm{U}$ and $\mathrm{V}$ in $\mathrm{G}$. In the second phase (cost distribution), it simply eliminate the non optimal link of the enclosed graph and the resulting graph is a minimum power topology, It consumes least power among the all path having directed paths from each sensor to the sink. Each sensor broadcasts its cost to its neighbours; the cost of the node is needed amount of minimum power for determining a direct path to the sink.

\section{Small minimum-energy communication network (SMECN):-}

MECN improved propose is a SMECN [11] routing protocol, SMECN cost of the link maintenance is lower than MECN and more energy efficiency. SMECN is also computationally less difficult than MECN. Which is a minimal graph is characterized with regard to the minimum energy property. This property mentions that every pair of sensor nodes associated with a circular region, there is an energy efficiency path between them. That is the path between every pair of sensors achieves smallest cost in the term of energy utilization. SMECN protocol broadcast a neighbour discovery message using some initial power $\mathrm{p}$ to discover its immediate neighbours in every pair of sensors, getting the response from all the nodes and checks the set of sensors that replied to a neighbours discovery message is the theoretical set of immediate neighbours. If that, the sensor nodes will communicate with its immediate neighbours using its corresponding power up. If not, it transmits with more power and rebroadcasts its neighbour discovery message.

Advantages and Disadvantages of location based protocol GAF, GEAR, MECN, and SMECN:-

1. Geographic Adaptive Fidelity (GAF)

Advantages:

1. Optimize the performance of WSN

2. Highly Scalable

3. Maximize the network lifetime

4. Limited energy conservation

Disadvantages:

1. High overhead

2. Doesn't take care of QoS during data transmission.

3. Limited mobility

4. Limited power management

2. Geographic and Energy Aware Routing (GEAR)

Advantages:

1. Increase the Network lifetime

2. Reduces Energy Consumption

Disadvantages:

1. Limited Scalability

2. Limited Mobility

3. Limited Power management

4. High overhead

5. Doesn't take care of QoS

\section{Minimum Energy Communication Network (MECN)}

Advantages:

1. Maintains energy network with low power

2. Fault tolerant

3. Optimal spanning

Disadvantages:

1. Fault tolerant depends upon specific application

2. Low scalability

3. Link repair required upon topology changes

\section{Small Minimum Energy Communication Network (SMECN)} Advantages:

1. Less Energy than MECN

2. Links maintenance cost is less 


\section{Disadvantages:}

1. Maximum power usage

2. No. of broadcast messages is large

3. Link repair required upon topology changes

\section{Conclusion:-}

In WSN, the sensor nodes have a restricted transmission variety, and their processing and capacity of storage as well as their energy resources also are confined. Routing protocols for wireless sensor networks are liable for maintaining the routes in the network, which might depend on the application and network architecture and it have to ensure reliable communication under these conditions. In this paper, we have surveyed four routing protocol primarily based on the area through deliberating numerous category criteria, consisting of location statistics, network lifetime and QOS requirements. For every those protocols, we have mentioned an advantages and disadvantages.

\section{References:-}

1. Shio Kumar Singh, M P Singh, and D K Singh: Routing Protocols in Wireless Sensor Networks -A Survey International Journal of Computer Science \& Engineering Survey (IJCSES) Vol.1, No.2, November 2010

2. Lakshmi et al., International Journal of Advanced Research in Computer Science and Software Engineering 5(4), April- 2015, pp. 663-667: Location-Based Routing Protocol in Wireless Sensor Network

3. Jamal N. Al-Karaki Ahmed E. Kamal: A Survey Routing Techniques in Wireless Sensor Networks

4. Y. Xu, J. Heidemann, D. Estrin, IGeography-informed Energy Conservation for Ad-hoc Routing," In Proceedings of the Seventh Annual ACM/IEEE International Conference on Mobile Computing and Networking 2001, pp. 70-84.

5. K. Akkaya and M. Younis, "A Survey of Routing Protocols in Wireless Sensor Networks, " in the Elsevier Ad Hoc Network Journal, Vol. 3/3 pp. 325-349, 2005.

6. I. Akyildiz, W. Su, Y. Sankarasubramaniam, and E. Cayirci, "A survey on sensor networks," IEEE Communications Magazine, Volume: 40 Issue: 8, pp.102-114, August 2002.

7. Ana Maria Popescu, Gabriel Ion Tudorache, Bo Peng, Andrew H. Kemp, "Surveying position based routing protocols for Wireless Sensor Networks" International Journal of Communication Networks and Information Security (IJCNIS) vol4, No1 page(s):41-65.

8. Arampatzis, Th, Lygeroes, J, Manises, S, "A Survey of Applications of Wireless Sensors and Wireless Sensor Networks", Intelligent Control, IEEE International Symposium on, Mediterrean Conference on Control and Automation, Page(s): 719 - 724, 2005.

9. Avni Kaushik, "A review on Routing Techniques in Wireless Sensor Networks", Vol. 3, Issue 6, June 2014

10. V. Rodoplu, T.H. Ming, "Minimum Energy Mobile Wireless Networks", IEEE Journal of Selected Areas in Communications 17 (8) (1999) 1333-1344.

11. L. Li., J.Y. Halpern, Minimum-energy mobile wireless networks revisited, Proc. IEEE International Conference on Communications (ICC), pages 278-283, June 2001 and extended version to appear in IEEE Transactions on Wireless Communications, 2003.

12. Y. Yu, R. Govindan, D. Estrin, Geographical and Energy Aware Routing: a recursive data dissemination protocol for wireless sensor networks, 2001

13. Shital Y. Agrawal, Prof. C. M. Raut," A Survey on Location Based Routing Protocols for Wireless Sensor Network", International Journal of Emerging Technology and Advanced Engineering ISSN 2250-2459, ISO 9001:2008 Certified Journal, Volume 3, Issue 9, September 2013 